\title{
Poésies orales chantées en France. Travaux récents
}

Yves Defrance

\section{(2) OpenEdition}

Journals

\section{Édition électronique}

URL : http://journals.openedition.org/ethnomusicologie/1675

ISSN : 2235-7688

\section{Éditeur}

ADEM - Ateliers d'ethnomusicologie

Édition imprimée

Date de publication : 1 janvier 1998

Pagination : 272-279

ISBN : 2-8257-0639-6

ISSN : 1662-372X

\section{Référence électronique}

Yves Defrance, "Poésies orales chantées en France. Travaux récents », Cahiers d'ethnomusicologie [En ligne], 11 | 1998, mis en ligne le 07 janvier 2012, consulté le 19 avril 2019. URL : http:// journals.openedition.org/ethnomusicologie/1675

Ce document a été généré automatiquement le 19 avril 2019.

Tous droits réservés 


\title{
Poésies orales chantées en France. Travaux récents
}

\author{
Yves Defrance
}

\section{RÉFÉRENCE}

Léon Froment : Chansons du pays d'Oc. Rodez : Editions du Rouergue, 1996. 350 p.

Carnets de route de Yann-Fanch Kemener. Morlaix : Skol Vreizh, 1996. 358 p., illustrations, transcriptions musicales, $2 \mathrm{CD}$

Gilles Goyat: Chansoniou eur Vigoudenn, Etude d'un répertoire. Brest : Emgleo Breir-Ar Skol Vrezoneg, 1997. 442 p., illustrations, cartes, transcriptions musicales Louisette RADIOYES : Traditions et chansons de Haute-Bretagne. Le répertoire de Saint-Congard et ses environs 1962-1970, 2 volumes. Aix-en-Provence : Edisud/CNRS Editions, 1995. 286 p., cartes, transcriptions musicales (vol.1) ; Redon : Groupement Culturel Breton du Pays de Vilaine, 1997. 384 p. (vol. 2)

1 Après l'engouement spectaculaire des années 1980 pour les musiques traditionnelles instrumentales régionales en France, manifesté notamment par les progrès importants dans la facture et le jeu des hautbois, cornemuses, vielles, violons et autres accordéons « diatoniques », la chanson traditionnelle semble faire un retour en force dans les années 1990. A cappella et en langue régionale, des chanteurs et chanteuses, souvent professionnels, font renaître le répertoire de la paghiella corse, de la basa Ahaideak basque, des chants occitans ou des gwerzioù bretons. Recueils de chansons et études linguistiques ou musicologiques, accompagnent ce mouvement. Nous proposons ici une première sélection de publications récentes.

2 Léon FROMENT : Chansons du pays d'Oc. Rodez: Editions du Rouergue, 1996. 350 p., transcriptions musicales.

A contre-courant de la tendance éditoriale française, les Editions du Rouergue, établies à Rodez, publient un ouvrage de compilation de chansons en langue occitane. Elles sont le fruit d'une collecte réalisée au début du $\mathrm{XX}^{\mathrm{e}}$ siècle par le musicien Léon Froment 
(1869-1934). Natif de l'Aude, il passa sa vie à diriger différents orphéons dans le Rouergue, département de l'Aveyron. Dans les années 1930, il publia Chansons du Rouergue, en deuxvolumes. Seules les chansons en langue d'oc sont ici reproduites, aux côtés de 46 chansons inédites, et de la réédition des Chants populaires du Bas-Quercy, publiés en 1889 à Paris par Emmanuel Soleville et patiemment recopiés dans un cahier par Léon Froment. $\mathrm{Au}$ total, 152 textes occitans, avec leur traduction française et leur mélodie en fac-simile. Commentaires et informations sommaires sur les conditions de collecte, accompagnent chaque chanson. Bien entendu, nous sommes en pleine période folkloriste de la IIIe République. Léon Froment, en harmoniste consacré, propose parfois un accompagnement de piano. Emmanuel Soleville, dont la publication correspond au centenaire de la Révolution française, reprend la classification thématique d'alors : légendes et ballades, noëls, berceuses, romances, idylles, saynètes, sérénades, aubades, chansons bouffonnes, airs de danse, farandoles.

Dénué de toute intention scientifique, ce document veut avant tout célébrer la langue occitane, descendant de celle des troubadours de l'époque médiévale, conservée oralement par des locuteurs majoritairement ruraux. Comme la jaquette le précise, Chansons du pays d'oc s'adresse principalement "à ceux qui, les soirs de fête, essaient vainement de retrouver les chants qu'ils entendirent enfants et qui restent muets au milieu d'une phrase ou d'une mélodie, joignant leurs « la, la, la ... » à ceux de leurs voisins dans un effort commun souvent infructueux ».

4 Carnets de route de Yann-Fanch Kemener. Morlaix : Skol Vreizh, 1996. 358 p., illustrations, transcriptions musicales, $2 \mathrm{CD}$.

L'invitation à chanter jaillit avec autant de ferveur dans l'ouvrage du chanteur professionnel Yann-Fanch Kemener qui, un siècle après Léon Froment, consacre, lui aussi, sa vie à la poésie populaire orale chantée, non pas en occitan, mais dans une autre langue régionale de France, sa langue maternelle: le breton. Ses Carnets de route livrent l'expérience exceptionnelle, probablement unique dans l'Europe de l'Ouest contemporaine, d'un jeune homme, né vers 1960 dans un milieu rural, culturellement protégé, où la tradition orale s'est maintenue beaucoup plus longtemps qu'ailleurs. Dans cette Haute-Cornouaille, désignée comme le cœur de la Bretagne (kalon vreizh), la langue bretonne, parlée spontanément, véhicule une littérature orale particulièrement riche et bien conservée. Avec une simplicité et une modestie peu communes, l'auteur expose les conditions dans lesquelles se fit son apprentissage du grand répertoire vocal celtique, qu'il interprète aujourd'hui sur toutes les scènes d'Europe avec le talent que l'on sait: « Ma grand-mère est morte en chantant. [...] Elle nous a dit : Je vais vous en chanter une autre ... et puis elle s'est éteinte comme ça. » «Voilà ce que me racontait ma mère quand j'étais enfant. Elle ajoutait encore : «Ma grand-mère avait beaucoup de caractère, mon grand-père savait regarder les étoiles, mon oncle Sulian, celui qui avait déserté à la Grande Guerre et dont on n'a jamais su ni quand, ni comment il était mort, grimpait dans les arbres quand il était jeune et se mettait à chanter, et tout le monde s'arrêtait de travailler pour l'écouter. Mon père, ma mère, mes oncles Pierre-Jean et Eugène, ma tante Marie-Louise étaient tous de grands chanteurs et de bons danseurs. Regarde aujourd'hui mon cousin Eugène Grenel comment il chante ... ».

5 Autodidacte en matière de collecte, mais doué d'un naturel capable de dérider les personnes les plus méfiantes, Yann-Fanch Kemener parcourt les campagnes les plus reculées de la Basse-Bretagne et réussit à enregistrer des dizaines de chansons, fortes d'un grand nombre de couplets (jusqu'à 47 ), aux versions inédites, voire inconnues. Un 
siècle et demi après La Villemarqué, le grand chanteur nous montre combien le petit peuple de Bretagne, parfois analphabète, souvent non francophone, sut conserver oralement un patrimoine inestimable. Songeons que la gwerz «Skolan», qu'il recueille auprès de Jean-Louis Rolland à Carhaix, en 1979, trouve des correspondances littéraires indéniables dans un manuscrit gallois $\mathrm{du} \mathrm{XI}^{\mathrm{e}}$ siècle et, par conséquent, en renvoie l'origine, avant l'émigration des Bretons insulaires vers l'Armorique, aux $\mathrm{VI}^{\mathrm{e}}$ et $\mathrm{VII}^{\mathrm{e}}$ siècles. Par son excellente connaissance du terrain, de la sensibilité de ses habitants, des subtilités dialectales du lieu, Yann-Fanch Kemener sauva in extremis des pans entiers du répertoire des gwerzioù, qu'il fit connaître lui-même dès les années 1970 dans ses interprétations publiques courageuses, a cappella et totalement sourdes aux modes folk d'alors. Car si bon nombre de collecteurs publièrent recueils et études, personne ne sut si bien que lui redonner vie à des textes centenaires muets, encore moins à des mélodies silencieuses, transcrites plus ou moins habilement. Ces Carnets de route retracent donc une vingtaine d'années de quête personnelle, guidée par une démarche d'interprète. C'est là toute sa valeur. L'auteur place au premier plan les chanteurs, avec lesquels il noua une amitié profonde qui transparaît à chaque page, notamment dans l'introduction, qu'il nomme lui-même « Mémoire du cœur ».

6 L'ouvrage se découpe en deux parties inégales. La première, forte de 85 pages, apporte de très nombreuses informations sur les enquêtes. On y trouve une biographie détaillée, parfois illustrée, de 57 chanteuses et chanteurs. Deux universitaires, le linguiste Francis Favereau et l'historien Joseph Rio, présentent chacun une étude relevant de leur spécialité. Poalig Combot dresse un tableau comparatif des versions « Kemener » et « La Villemarqué ». André Le Meut, sonneur de bombarde et chef d'un des trois meilleurs bagadoù de Bretagne, réalisa les transcriptions musicales. Il expose son point de vue sur «la souplesse rythmique : un élément magique du chant». Un petit lexique des termes utilisés pour le chant, la poésie, la musique et la danse complète utilement cette présentation richement documentée. Suivent 166 chants en langue bretonne, avec leur traduction française, classés par genre littéraire : 1 . Chansons d'inspiration religieuse ou concernant des événements surnaturels ; 2 . Chants d'amour, de mariage et de deuil ; 3. Chansons de clercs ; 4 . Les métiers ; 5 . Chansons de conscrits, de soldats et de guerre ; 6 . Enlèvements et meurtres ; 7. Divers.

7 Si les textes présentent un intérêt de première importance, les transcriptions musicales, bien que très soignées en comparaison avec les recueils de chants populaires auxquels nous sommes habitués, laissent encore parfois à désirer. Pourquoi placer une armure lorsqu'une mélodie n'évolue que sur quelques degrés? Ceci oriente à tort vers une interprétation tonale, alors qu'une proportion importante des mélodies relève d'échelles non heptatoniques, souvent pentatoniques. A l'inverse, pourquoi mettre un bémol à la clé, pour ensuite placer un bécarre devant chaque note si? Le découpage rythmique paraît plus convaincant, quoique la présence de mesures oblige le transcripteur à opérer des choix un peu arbitraires. De plus, l'absence d'indication de tempo risque d'induire en erreur de nombreux lecteurs. C'est tout le problème de la transcription qui rejaillit. Conscient que celle-ci reste un moyen de lecture rapide, nous n'en ferons pas le procès point par point. D'autant que deux disques compacts viennent illustrer utilement le propos. Fait unique, à notre connaissance, nous pouvons entendre des entretiens d'enquête en langue bretonne entre le collecteur et les informateurs. Ces exemples parlés et chantés ont pour vertu de plonger l'auditeur dans l'univers linguistique et musical d'une tradition orale vivante, et pourtant si lointaine pour un non-bretonnant. 
Dans ses Carnets de route, Yann-Fanch Kemener découvre un répertoire inédit qui, par la variété et la qualité des exemples, sonores ou transcrits, séduira amateurs et spécialistes de poésies populaires orales. Il apporte surtout un témoignage irremplaçable sur l'ultime phase d'une civilisation paysanne préindustrielle basée sur l'oralité, dont les modes de production ont définitivement disparus au cours du $\mathrm{XX}^{\mathrm{e}}$ siècle dans une Europe déjà partiellement postindustrielle. Grâce à son action, suivie par d'autres, la tradition vocale bretonne a quitté les champs et les chaumières, mais elle trouve d'autres lieux d'expression et de renouvellement, adaptés au monde d'aujourd'hui.

9 Gilles GOYAT : Chansoniou eur Vigoudenn, Etude d'un répertoire. Brest : Emgleo Breir-Ar Skol Vrezoneg, 1997. 442 p., illustrations, cartes, transcriptions musicales.

Avec Gilles Goyat nous quittons la Haute-Cornouaille, mais pas la Bretagne. Il propose une analyse linguistique très poussée sur le répertoire d'une chanteuse d'un autre terroir : le Pays Bigouden. Natif du village jouxtant celui du célèbre écrivain Per-Jakez Hélias, l'auteur pratique la langue bretonne depuis son enfance et maitrise parfaitement les subtilités du dialecte bigouden. Bon musicien amateur, il s'est naturellement intéressé aux poésies orales populaires et c'est dans le cadre d'un travail universitaire qu'il réalisa cette étude. Dix-sept chants, enregistrés auprès de Catherine Madec en 1964-1965 par Donatien Laurent (enquête CNRS pluridisciplinaire de Plozévet) sont transcrits avec leur mélodie, traduits en français, et font l'objet d'une analyse fort intéressante. Celle-ci porte sur six points principaux : thème littéraire, récit, versification, particularités de la langue utilisée, rapport entre les paroles et la mélodie, comparaisons avec d'autres versions (bretonne, françaises voire autres). Suit une présentation complète du système phonologique du breton parlé de Plozévet.

10 Au-delà de l'apport de premier ordre aux recherches linguistiques et dialectales, ces Chansons d'une Bigoudène présentent l'intérêt de soulever des questions dans un domaine assez peu exploré par les ethnomusicologues : quelles relations entretiennent prosodie littéraire et ce que l'auteur nomme "prosodie musicale»? En complément des judicieuses remarques qui accompagnent chaque chant, le court et dense chapitre traitant de la prosodie musicale mérite toute notre attention. Gilles Goyat, s'appuyant sur des faits linguistiques irréfutables - l'informatrice, née en 1894 était monolingue - met ses compétences au service d'une réflexion sur la concordance entre l'accentuation du texte parlé et l'accentuation de la mélodie qui le supporte, dans les dix-sept cas examinés. Ainsi distingue-t-il deux types de syllabes parlées (accentuée et inaccentuée) et quatre types de syllabes chantées, à savoir: brève accentuée, longue accentuée, brève inaccentuée et longue inaccentuée. Des schémas accentuels sont alors établis à partir des mélodies classées selon la longueur des vers qu'elles supportent $(6,7,8,12,13,14,15$ pieds). Il apparaît que, dans toutes les mélodies supportant des vers à hémistiches asymétriques, une note accentuée termine l'hémistiche court tandis que l'hémistiche long connaît une avant dernière note accentuée et une dernière note non accentuée. Puis, Gilles Goyat superpose l'accentuation de la langue parlée, non emphatique, et celle imposée aux paroles chantées par la mélodie. Son regard se fixe plus précisément en début de vers, à la césure et à la rime. Les cas particuliers, diérèses, mélismes, chevilles et élisions ne sont pas omis.

An hini a goll ar zon a goll an ton, "celui qui perd ses mots perd son air ", dit le proverbe breton. Le cas d'une poésie chantée permet d'observer dans le détail les influences des paroles sur la mélodie, notamment dans le cas d'un vers irrégulier ou d'une syllabe franchement accentuée dans la langue parlée. Pourtant, ce sont toujours « les débuts ou 
les parties centrales des cellules mélodiques qui sont transformés, jamais les parties finales qui se comportent comme des piliers inamovibles sur lesquels repose l'édifice mélodique. Ce sont les notes de 'passage' ou notes de 'liaison' qui sont affectées, alors que les notes d"appui' ou notes 'principales' restent inchangées». Mais, la plupart du temps "c'est la mélodie qui impose son accentuation aux paroles. Ainsi, lorsqu'une syllabe inaccentuée dans la langue parlée tombe sur un temps ou une partie de temps musicalement fort, la syllabe chantée est accentuée ; dans ce cas c'est l'accent musical qui l'emporte sur l'accent de la langue». (pp. 265-266). Bien que la prépondérance des paroles sur les mélodies soit nette, lorsqu'il y a discordance, c'est la mélodie qui impose son accentuation aux paroles. Ces conclusions, étayées par une connaissance profonde des grandes collectes du répertoire breton d'expression celtique, viennent contredire, ou tout au moins nettement atténuer, les assertions du musicologue Bourgault-Ducoudray, professeur d'analyse et d'histoire de la musique au Conservatoire National Supérieur de Musique de Paris, qui, à la fin du XIX siècle, s'était livré à une importante collecte de chants populaires en Grèce, puis en Bretagne, et s'en était largement inspiré pour élaborer de nouvelles théories musicales, aujourd'hui stériles. Voilà une analyse fine qui pourrait servir de modèle opératoire, simple mais efficace, dans l'appréhension du complexe relationnel parole/musique.

Louisette RADIOYES : Traditions et chansons de Haute-Bretagne. Le répertoire de SaintCongardet ses environs 1962-1970, 2 volumes. Aix-en-Provence: Edisud/CNRS Editions, 1995. 286 p., cartes, transcriptions musicales(vol.1) ; Redon : Groupement Culturel Breton du Pays de Vilaine, 1997. 384 p. (vol. 2).

Le titre est sans équivoque : il s'agit bien d'une monographie de village, et d'une qualité rare. Louisette Radioyes, native du lieu, s'est livrée à une étude à la fois externe et interne, où l'observation participante porte tous ses fruits. La petite commune rurale de Saint-Congard est située dans la partie orientale du département du Morbihan et ses habitants parlent le gallo, un dialecte roman légèrement teinté de termes et d'expressions celtiques. Pour autant, la quasi totalité du répertoire vocal recueilli et transcrit ici n'en est pas moins en français. C'est que nous avons affaire à cette Haute-Bretagne romanisée, dont les habitants d'aujourd'hui, complexés d'avoir " perdu » la langue bretonne, depuis le XII ${ }^{e}$ siècle, se désignent comme "sots Bretons", auto-dénigrement lourd de conséquences dans la construction de leur identité. On pense inévitablement à Drâgus, village roumain des Carpates, dont Constantin Brâiloiu rend compte après une enquête échelonnée de 1929 à 1932. Mais, dans le cas gallo, le phénomène d'interférence entre l'écrit et l'oral, si habituel dans l'histoire des peuples d'Europe occidentale, s'avère plus aisément repérable.

L'auteur fournit un important corpus de près de 500 chansons et formules chantées, dont environ 300 à texte de plusieurs strophes, avec la notation de leur «mélodie-type ». Et ceci sur un rayon de cinq kilomètres à peine! C'est souligner le caractère exceptionnel d'une telle moisson, et ce à une époque toute récente. Quand on sait combien il devenait difficile d'entendre des chansons traditionnelles en situation dans la France rurale dès les lendemains de la Première Guerre Mondiale, le travail de Louisette Radioyes n'en est que plus remarquable. Pour avoir enquêté sur ce même terrain, très exactement dix ans plus tard, je puis personnellement témoigner de la qualité tant littéraire que musicale du matériau présenté ici. La commune de Saint-Congard et les quatre communes voisines sur lesquelles ont été réalisés les entretiens et enregistrements offrirent à Louisette Radioyes 
la possibilité d'observer in situ des faits qui, dix ans plus tard, étaient déjà relégués dans le passé.

14 Au-delà de l'intérêt évident du corpus et des perspectives comparatistes qu'il peut offrir aux folkloristes du domaine francophone, héritiers des travaux de Conrad Laforte et, plus encore, de Patrice Coireault, ce qu'apporte Louisette Radioyes tient surtout, à mon sens, dans l'approche sensible du répertoire. Celui-ci est toujours présenté dans une connexion étroite avec son milieu, mettant ainsi en évidence un devenir solidaire. Les informations réunies sur la façon dont ce terroir est passé, entre 1910 et 1970, d'un état à un autre sont particulièrement précieuses. Très peu de chercheurs sont arrivés à rendre aussi bien et aussi modestement ce que fut la chanson traditionnelle dans les campagnes françaises jusqu'aux bouleversements économiques des $\mathrm{XIX}^{\mathrm{e}}$ et $\mathrm{XX}^{\mathrm{e}}$ siècles qui en précipitèrent la chute. Le travail, circonscrit à une superficie géographique ne dépassant pas les $50 \mathrm{~km}^{2}$, présente toutes les garanties de sérieux et de précision (classification analytique, index des incipit, index analytique, glossaire gallo, liste des informateurs, bonne bibliographie). En outre, un court commentaire accompagne chaque chanson et le lecteur a droit à des précisions sur une centaine d'informateurs dont la moitié font l'objet d'une courte biographie, parfois accompagnée d'une photographie. Mais, plus qu'un simple catalogue raisonné, cet ouvrage fera date dans l'histoire de l'ethnomusicologie du domaine français pour avoir apporté un regard de l'intérieur sur les mille et une subtilités qui firent de la chanson traditionnelle un acte quotidien complètement imbriqué dans la vie de ceux qui en firent usage. Avec la complicité des habitants de Saint-Congard, l'auteur nous plonge dans l'univers de ce que fut leur vie rurale, en quasi autarcie durant des siècles. La localisation étroite de l'enquête lui permit de rechercher très en profondeur dans la mémoire encore vivante de sa parentèle, de ses voisins, amis et relations proches. Qu'on songe que cette petite société tournée sur elle-même, où l'endogamie, parfois étroite, est pratique courante, ne connaîtra des routes empierrées qu'entre les deux guerres mondiales! Dans cette collectivité d'interconnaissance, de type holiste, selon la définition de Louis Dumont, le chant occupe une grande place et ce à chaque moment de la journée. Tout le monde chante, tout le temps et en toute occasion. La musique instrumentale tient si peu de place - en la personne d'un sonnou ( = joueur d'accordéon) - que les formules de mémorisation des airs à danser utilisent toutes les incipit des textes qu'ils supportent. Indéniablement, la danse traditionnelle de ce terroir gallo ne peut se penser sans le chant, désigné ici comme son "âme même ». Louisette Radioyes a d'ailleurs judicieusement intitulée la première partie de sa passionnante présentation « Un pays de chanteurs ». Le chapitre consacré à la musique dans la vie est particulièrement réussi. Mais le plus remarquable tient sans doute à la façon dont l'auteur présente les façons de chanter, les styles vocaux, l'apprentissage des chansons, pour montrer à quel point les conditions d'existence des ces chansons sont liées à la vie communautaire. Tout semble se dire, se vivre à travers les chansons, mode de communication aux fonctions sociales multiples. L'individu n'existe pas sans les chansons qui le font exprimer ses sentiments, ses émotions circonstancielles, événementielles ou imprévues. "A chaque mot sa chanson ", me disait, en 1974, Jeanette Maquignon, informatrice de Ruffiac, communue limitrophe à Saint-Congard. Ceci traduisait bien l'un des usages du chant, parfois seul capable de codifier le social dans un milieu traditionnel, car le chant, en particulier le chant alterné collectif, peut certes être " source gratuite de plaisir et de beauté », mais aussi « facteur de solennisation ", « composant nécessaire d'un rituel », « forme donnée à de nombreuses activités sociales ». 
15 Contrairement aux assertions de la majorité des revivalistes d'aujourd'hui, il apparait que le chant traditionnel ne s'exécute que très rarement durant les moments de repos ou de loisir (le cliché du brave paysan, gai luron dans ses chansons un peu lestes, relève d'un folklore d'opérette, hélas encore très tenace). Bien au contraire, sa fonction dynamogénique en fait une discipline de travail et c'est toujours en situation qu'il prend tout son sens. Quand disparaissent la garde des troupeaux, les veillées (teillage du chanvre, filage...), le labour aux bœufs, les quêtes saisonnières, ce sont des pans entiers du répertoire vocal qui cessent de s'actualiser et d'avoir des raisons de se transmettre. Comme le souligne justement Jean-Michel Guilcher dans sa préface: «ce n'est pas seulement la vie d'un milieu qu'elles (les chansons) aident à mieux comprendre. C'est le fonctionnement de la Tradition, avec le mouvement qui anime son apparente inertie ». 